\title{
Comparison of the results of tracer experiments in lakes with predictions based on horizontal mixing models
}

\author{
F. Peeters, A. Wüest and D. M. Imboden
}

\section{Introduction}

Mixing processes play a major role in the distribution of dissolved substances and particles, including plankton, in natural waters. In lakes, vertical mixing has been studied extensively, while studies of horizontal mixing are rare. Lakes are commonly assumed to be horizontally homogeneous, and consequently horizontal mixing is frequently neglected. However, its effects can be of great importance to processes occurring at the lake boundaries and to water pollution studies, since horizontal turbulent diffusion is important in transporting substances over great distances and in diluting high concentrations caused for example by accidental pollution events.

Up until now, horizontal mixing has been investigated mainly in very large water bodies (e.g. in the ocean by OKuBo 1971 and in Lake Ontario by MuRThy 1976), or in very small lakes (e.g. by QuAY et al. 1979). With the objective of obtaining a better understanding of relative diffusion (diffusion relative to the centre of mass) in the hypolimnion of medium-sized lakes (approx. $25 \mathrm{~km}^{2}$ ), three tracer experiments were conducted in the course of this research. In the following section the experimental technique and the method of data reduction is described very briefly (for a detailed description see PEETERS \& WUEST 1992). Subsequently, the experimental results are presented and compared with MuRTHY's (1976) Lake Ontario data and with the ocean data of Oxuno (1971) and Ewart \& BENDINER (1981). This is followed by a short discussion of the applicability of some models describing horizontal diffusion.

\section{Method}

The method employed here by us to study horizontal transport mechanisms involved observation of the development of the concentration distribution of a tracer following its point release in the hypolimnion of a lake. As tracer we used the fluorescent dye uranin (sodium fluorescein), the concentration of which can be measured quasi-continously with a fluorometer at rates of about $10 \mathrm{~Hz}$. A commercial CTD sonde attached to the fluorometer provided information on its depth, as well as on the temperature and electrical conductivity of the water at that depth, at a rate of $8 \mathrm{~Hz}$. A positioning system gave the $\mathrm{x}$ and $\mathrm{y}$ coordinates of the ship with a resolution of $\pm 1 \mathrm{~m}$ at a rate of $1 \mathrm{~Hz}$. On-line data display enabled an efficient survey of the tracer distribution. For each tracer experiment, the setup allowed the tracer distribution to be determined up to seven times in the course of 3 days. Each survey of the "tracer cloud" consisted of about 100 vertical profiles, from which the total mass and centre of mass of the cloud along with the variances and covariances of the tracer distribution could be calculated. Each vertical profile was integrated, and the resulting horizontal array of vertically integrated concentrations $\mathrm{C}(\mathrm{x}, \mathrm{y})$ was interpolated horizontally to obtain iso-concentration lines (Fig. 1) and to enable further integration in the $\mathrm{x}, \mathrm{y}$ directions. The coordinates of the centre of mass $\left(x_{s}, y_{s}\right)$, the variances $\left(\sigma_{x}^{2}\right.$ and $\left.\sigma_{y}^{2}\right)$ and the covariance $\left(\sigma_{\mathrm{xy}}\right)$ are given by:

$$
\begin{aligned}
& x_{s}=\frac{1}{M} \iint x C(x, y) d x d y \\
& y_{s}=\frac{1}{M} \iint y C(x, y) d x d y \\
& \sigma_{x}^{2}=\frac{1}{M} \iint\left(x-x_{s}\right)^{2} C(x, y) d x d y \\
& \sigma_{y}^{2}=\frac{1}{M} \iint\left(y-y_{s}\right)^{2} C(x, y) d x d y \\
& \sigma_{x y}=\frac{1}{M} \iint\left(x-x_{s}\right)\left(y-y_{s}\right) C(x, y) d x d y
\end{aligned}
$$

where $M$ represents the integrated (total) mass of the tracer.

From these results, the variances $\sigma_{1}^{2}$ and $\sigma_{s}^{2}$ along the major and minor principal axes, respectively, of the tracer cloud can be easily obtained. We define the size of a tracer cloud as $\sigma_{r}^{2}=2 \sigma_{l} \sigma_{s}$. For a two-dimensional Gaussian distribution, $\pi \sigma^{2}$ corresponds to $63 \%$ of the total integral. 


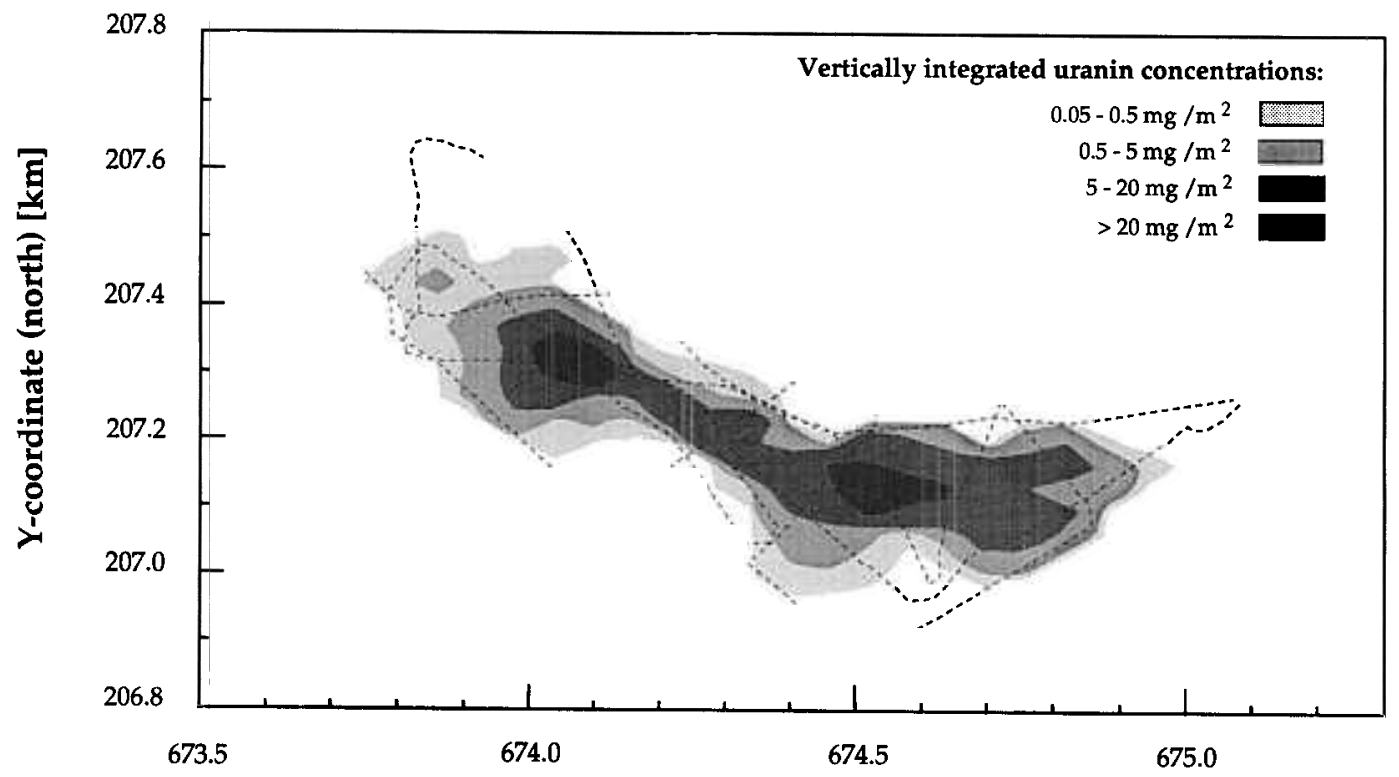

$X$-coordinate (east) $[\mathrm{km}]$

Fig. 1. Uranin distribution 30 hours after its point release (at $18 \mathrm{~m}$ depth) into Vitznau basin on 25 March 1991. The dashed line represents the path of the ship during the survey of the tracer cloud.

\section{Results}

The development in time of a tracer distribution starting from a single point release is shown in Fig. 2. Advection is responsible for the change in position of the centre of mass (symbol X), while diffusion is responsible for the increase in the variances along the minor and major principal axes (the axes of the ellipses shown in Fig. 2 correspond to $3 \sigma_{\mathrm{I}}$ and $3 \sigma_{s}$, respectively). The tracer distributions measured were not radially symmetric (Figs. 1 and 2). The increase in cloud sizes calculated from experiments in two basins of Lake Lucerne (Vitznau basin and Urner See) are illustrated in Fig. 3. Each symbol represents a unique experiment, i.e. the measurements following a single tracer release. In the three Lake Lucerne experiments, the growth in cloud size with time appears to be approximately proportional to elapsed time to the power 1.1, 1.3 and 1.5 , respectively. The difference between the three curves corresponding to these three experiments in Fig. 3 is due to differences in environmental conditions. Data from individual experiments in the ocean and in Lake Ontario are in good agreement with these results (Fig. 3).

In the literature (OKuBo 1971, MURTHY 1976), each cloud size measured is commonly regarded as

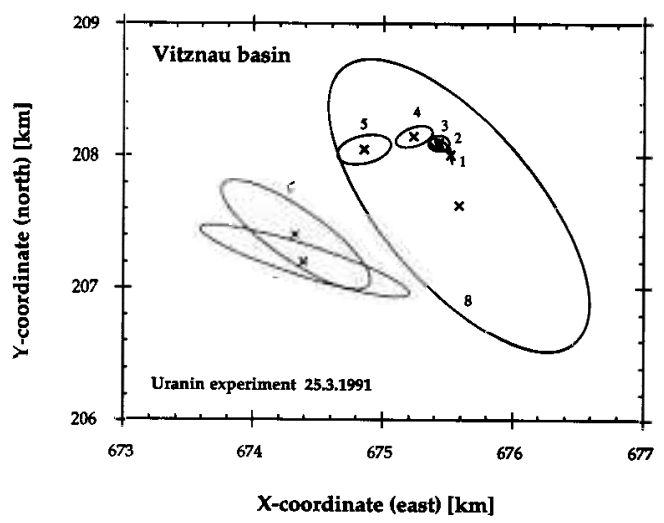

Fig. 2. Development in time of a tracer distribution in Vitznau basin. Assuming the tracer distribution to be Gaussian, the ellipse perimeters shown represent $1 \%$ of the maximum concentration of the particular cloud concerned. $99 \%$ of the total mass of each tracer cloud lies within the perimeter of the corresponding ellipse. The symbol $\mathrm{X}$ represents the centre of mass of each of the tracer clouds.

the result of an independent experiment. If it is assumed that environmental conditions are the same for all experiments, all cloud sizes determined can be combined into a single experiment. De- 


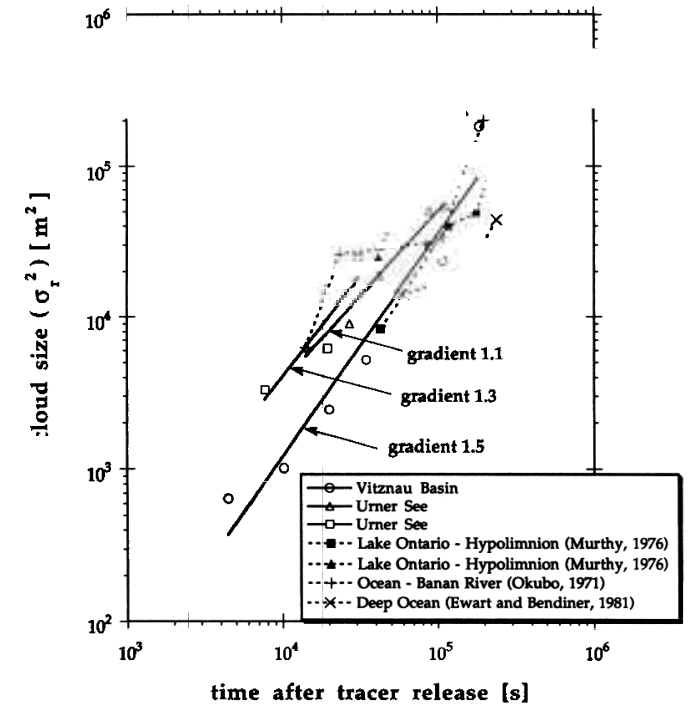

Fig. 3. Cloud size as a function of time in lakes and in the ocean. The solid line represents a power law fit to measurements of cloud size conducted in the Vitznau basin in March $1991(0)$, and in Urner See in September $1991\left(^{\circ}\right)$ and October $1991(\Delta)$. The dashed line connects data points from cloud size measurements obtained by MURTHY (1976) in two experiments in the hypolimnion of Lake Ontario $(s, n)$, and data points from cloud size measurements conducted in the ocean by Orubo $(1971)(+)$ and by Ewart $\& \tau$ BENDINER (1981) (X).

viations from a particular fit to the data (e.g. a power law) using such an ensemble of experiments are then regarded as statistically random. However, the development of the tracer distribution in time in experiments taken separately does not necessarily agree with the time dependence obtained when the experiments are combined. In the ocean (Orubo 1971) and in Lake Ontario (MuRTHY 1975), experimental models obtained by fitting an ensemble of experiments to a power law predict cloud size to increase with time according to $\sigma_{\mathrm{r}}^{2} \sim \mathrm{t}^{2.3}$ and $\sigma_{\mathrm{r}}{ }^{2} \sim \mathrm{t}^{3.3}$, respectively. These experimental models neither agree with the measurements from our individual experiments nor do they give a good description of individual experiments in the ocean or in Lake Ontario, at least for the scales relevant to our work (Fig. 3). The number of individual experiments, as well as the number of times cloud size is measured during each experiment, appears to influence the behaviour of such "ensemble models". Theoretical horizontal diffusion models should therefore be tested using individual experiments, not ensembles.
Effective horizontal diffusion coefficients $K_{\text {eff }}$ can be calculated from cloud sizes using the relationship $\mathrm{K}_{\mathrm{eff}}=\frac{1}{4} \sigma_{\mathrm{r}}^{2} \mathrm{t}^{-1}$. In our experiments, values of $\mathrm{K}_{\mathrm{eff}}$ increase with cloud size and typically range between 0.03 and $0.3 \mathrm{~m}^{2} \mathrm{~s}^{-1}$. Effective horizontal diffusion coefficients are thus about to five orders of magnitude larger than turbulent vertical diffusion coefficients (typically of the order $10^{-6}$. $\mathrm{m}^{2} \cdot \mathrm{s}^{-1}$ to $\left.10^{-5} \cdot \mathrm{m}^{2} \cdot \mathrm{s}^{-1}\right)$. This is in agreement with measurements in very small lakes $\left(0.05 \mathrm{~km}^{2}\right)$ made by QuAY et al. (1979). Furthermore, in these small lakes $\mathrm{K}_{\mathbf{x}}$ was calculated to be $0.016 \cdot \mathrm{m}^{2} \cdot \mathrm{s}^{-1}$ (QUAY et al.) which is of the same order as in our experiments.

\section{Models}

Several models have been proposed for the description of horizontal diffusion in natural waters (e.g. Batchelor 1950, Okubo 1967, 1968 and 1971, Kuluenderg 1972, Young et al. 1982).

The inertial subrange model discussed by BATCHELOR (1950) is based on the following reasoning. Eddies significantly larger than the size of a tracer cloud cause advection of the centre of mass, while eddies of size equal to or smaller than that of the tracer cloud result in relative diffusion. The number of eddies contributing to the diffusion process depends on the cloud size; consequently, the larger the cloud is, the faster it grows. The growth rate depends, therefore, on the eddy size-scale distribution. Assuming steady, isotropic and homogeneous turbulence, and further assuming the cloud sizes to lie within the inertial subrange of the turbulence spectrum (where energy is transferred from larger to smaller eddies without external energy input and without dissipation of energy), the growth of cloud size with time is given by similarity arguments to be $\sigma_{\mathrm{r}}{ }^{2} \sim \mathrm{t}^{3}$. Clouds have to be radially symmetric since isotropic and homogeneous conditions have been presumed. However, this is usually not the case (Figs. 1 and 2) and cloud size increases more slowly than the third power of elapsed time (Fig. 3).

The model commonly used for vertical diffusion, the Fickian law, predicts an increase in cloud size according to $\sigma_{\mathrm{r}}{ }^{2} \sim \mathrm{t}$. Similar to the inertial subrange model described above, clouds must be radially symmetric, which, as discussed above, is not in agreement with the data.

Non-radially symmetric tracer distributions can be described by shear diffusion models (e.g. OKuBo 1967 and 1968, Kullenberg 1972, Young et al. 1982). Such models are based on the interaction 
between small-scale (Fickian) diffusion and the velocity field. Predictions of cloud sizes and variances along the principal axes of the tracer distribution depend on the velocity field. Since it is difficult to obtain detailed information on the velocity field experimentally, we used, as a first approximation, a model based on constant shear in the $y$ and $z$ directions (Oкиво 1968). The velocity field has the form $\mathrm{u}=\mathrm{y} \delta \mathrm{u} / \delta \mathrm{y}+\mathrm{z} \delta \mathrm{u} / \delta \mathrm{z}$ and $\mathrm{v}=\mathrm{w}=0$, where $u, v$ and $w$ represent the velocities in the $x$, $\mathrm{y}$ and $\mathrm{z}$ directions, respectively, and the shears $\delta \mathrm{u} / \delta \mathrm{y}$ and $\delta \mathrm{u} / \delta \mathrm{z}$ are constant. For times long after tracer release the principal axes of the tracer cloud should be along the direction of flow and perpendicular to it. Assuming that $K_{x}=K_{y}$, where $K_{x}$ and $\mathrm{K}_{\mathrm{y}}$ are the diffusion coefficients in the $\mathrm{x}$ and $\mathrm{y}$ di- rections, one obtains, using the techniques employed by Orubo (1967):

$$
\sigma_{\mathrm{r}}^{2}=2 \sqrt{4 \mathrm{~K}_{\mathrm{x}}^{2} \mathrm{t}^{2}+\frac{1}{3} \mathrm{~K}_{\mathrm{x}}\left[\left(\frac{\delta \mathrm{u}}{\delta \mathrm{y}}\right)^{2} \mathrm{~K}_{\mathrm{x}}+4\left(\frac{\delta \mathrm{u}}{\delta \mathrm{y}}\right)^{2} \mathrm{~K}_{\mathrm{z}}\right] \mathrm{t}^{4}}
$$

where $K_{z}$ represents the vertical diffusion coefficient. The first term under the square root sign is commonly neglected and discussion usually focuses on the second term. However, in order to test the shear model we considered all terms. Fits to the data based on the two independent parameters $\mathrm{K}_{\mathrm{x}}$ and $\mathrm{K}_{\mathrm{x}}(\delta \mathrm{u} / \delta \mathrm{y})^{2}+4(\delta \mathrm{u} / \delta \mathrm{z})^{2} \mathrm{~K}_{\mathrm{z}}$ were obtained using a least squares method. Fig. 4 shows the results of fitting our Lake Lucerne data, MURTHY's (1976) Lake Ontario data, and OKuBo's (1971) ocean data. The agreement between the

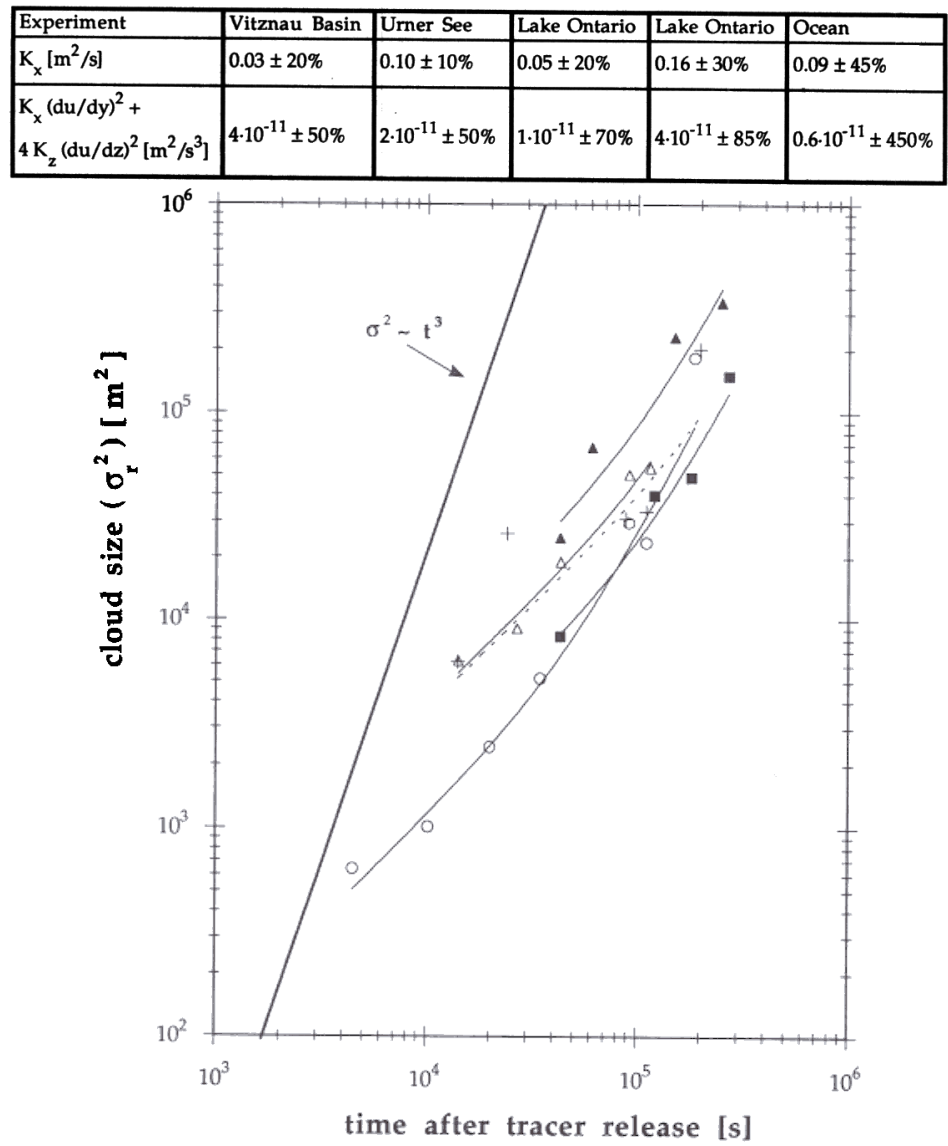

Fig. 4. Shear-diffusion model of horizontal diffusion fitted to experimental data. Fits to our data and to the data of MuRTHY (1976) are represented by solid lines; fits to ORUBO's (1971) data by a dashed line (for other symbols, see figure 3). The thick solid line indicates the growth of cloud size with elapsed time to the power 3 as predicted by BATCHELOR's (1950) turbulent diffusion model and (approximately) by OxuBo's (1971) and MURTHY's (1976) empirical models. 
data and the fitted curves (Fig. 4) suggests that the shear model provides a good description of the growth of tracer patches with time in mediumsized lake basins such as those of Lake Lucerne as well as in the considerably larger Lake Ontario. The model even seems to hold for experiments in the ocean, although data scatter is considerable. The results for the fit parameters are shown in Fig. 4. Values for $K_{z}$ in open lake waters are typically of the order $10^{-6} \cdot \mathrm{m}^{2} \cdot \mathrm{s}^{-1}$. Values on the order of $10^{-3} \cdot \mathrm{s}^{-1}$ for the shear $\delta \mathrm{u} / \delta \mathrm{z}$ were determined from drifters and moored current meters employed during the tracer experiments. Thus 4 $(\delta \mathrm{u} / \delta \mathrm{z})^{2} \mathrm{~K}_{\mathrm{z}}$ is of the order $4 \cdot 10^{-12} \cdot \mathrm{m}^{2} \cdot \mathrm{s}^{-3}$. Since the first fit parameter $\left(K_{x}\right)$ is of the order $10^{-}$ ${ }^{1} \cdot \mathrm{m}^{2} \cdot \mathrm{s}^{-1}$, the value of the second fit parameter $\left(\mathrm{K}_{\mathrm{x}}(\delta \mathrm{u} / \delta \mathrm{y})^{2}+4(\delta \mathrm{u} / \delta \mathrm{z})^{2} \mathrm{~K}_{\mathrm{z}}\right)$, i.e. $10^{-11} \cdot \mathrm{m}^{2} \cdot \mathrm{s}^{-3}$, seems to be reasonable as long as the horizontal shear $(\delta \mathrm{u} / \delta \mathrm{y})$ does not exceed $10^{-5} \cdot \mathrm{s}^{-1}$. The values for $K_{x}$ also seem to be plausible, as they are sufficiently large to guarantee nearly homogeous conditions in the lake, but are small enough to allow for a horizontally inhomogeous distribution of ${ }^{222} \mathrm{Rn}$ in lakes (IMBODEN \& JOLIER 1984). However, the data sets are very small and the purpose of the fits is not to determine the values of the model parameters, but to demonstrate the qualitative agreement between shear model and data. Finally, it should be mentioned that the requirement of point release set by all models discussed here could not be met. For the data sets from the Vitznau basin and Urner See, initial cloud sizes were approximately $100 \mathrm{~m}^{2}$ and $10 \mathrm{~m}^{2}$, respectively. This fact is of particular importance to the development of tracer distributions during the period shortly after release of the tracer. Since the determination of $K_{\mathbf{x}}$ depends on cloud size during this period, results must be interpreted carefully.

\section{Conclusions}

The results of experiments conducted by us in Lake Lucerne agree well with the results of experiments conducted in the ocean by Окиво (1971) and in Lake Ontario by MurThy (1976). Furthermore, effective horizontal diffusion coefficients are of the same order of magnitude as those determined in very small lakes by QunY et al. (1979). Thus, effective horizontal diffusion appears to be more or less independent of basin size.

Although the empirical power law models of Оxuвo (1971) and MURTHY (1976) agree with their data when applied to an ensemble of experiments, they do not agree with the data from individual experiments for cloud sizes within the range $5 \cdot 10^{2} \mathrm{~m}^{2}$ to $5 \cdot 10^{5} \mathrm{~m}^{2}$ (Fig. 4). In view of this discrepancy, tests of horizontal diffu- sion models should preferably be based on individual tracer experiments rather than an ensemble of experiments.

Based on our data from Lake Lucerne, on MuRTHY's (1976) data from Lake Ontario and on OruBo's (1971) ocean data, a shear-diffusion model with constant shear in $y$ and $z$ directions (OKuBo 1968) appears to provide a better description of the horizontal spreading of a tracer in time (for cloud sizes up to $5 \cdot 10^{5} \mathrm{~m}^{2}$ ) than the inertial subrange model applied by OruBo (1971) and by MuRTHY (1976). In order to provide further support for this hypothesis, additional tracer experiments in Lake Lucerne are being conducted.

\section{Summary}

Experiments in Lake Lucerne indicate growth of tracer patches to be approximately proportional to elapsed time raised to the power 1.3. Published data from Lake Ontario can be interpreted to be in agreement with this result. This suggests that an inertial subrange model of horizontal diffusion, such as that commonly used in the ocean, does not apply in lakes. A shear-diffusion model including small-scale (Fickian) diffusion appears to provide a better prediction of patch growth and patch asymmetries.

\section{Acknowledgements}

The authors would like to express their thanks to $M$. Schurter and P. Perrisset for their excellent technical support, to D. M. Livingstone for improving the English text and to G. PIEPKe for his valuable suggestions and advice in software development. The fluorometer was generously provided by the limnology group of the Federal Institute for Water Resources and Water Pollution Control (EAWAG). Financial support for this research was made available by the $S$ wiss National Science Foundation (grant 20-27751.89).

\section{References}

BATCHELOR, G. K., 1950: The application of the similarity theory of turbulence to atmospheric diffusion. - Q. II. R. Met. Soc. 76: 133-146.

Ewart, T. E. \& BeNDINER, W. P., 1981: An observation of the horizontal and vertical diffusion of a passive tracer in the deep ocean. - J. Geophys. Res. 86: $10974-10982$.

ImbOden, D. M. \& Joller, T., 1984: Turbulent mixing in the hypolimnion of Baldeggersee (Switzerland) traced by natural radon-222. - Limnol. Oceanogr. 29: $831-844$.

KULLENBERG, G., 1972: Apparent horizontal diffusion in stratified vertical shear flow. - Tellus 24: 17-28.

Murthr, C. R., 1976: Horizontal diffusion characteristics in Lake Ontario. - J. Phys. Oceanogr. 6: 7684. 
OxuBo, A., 1967: The effect of shear in an oscillatory current on horizontal diffusion from an instantaneous source. - Oceanol. Limnol. 1: 194-204.

- 1968: Some remarks on the importance of the "shear effect" on horizontal diffusion. - J. Ocea. nogr. Soc. Japan 24: 60-69.

- 1971: Oceanic diffusion diagrams. - Deep-Sea Res. 18: $789-802$.

Peeters, F. \& Wütst, A., 1992: Meß-System zur Erfassung dreidimensionaler Tracerverteilungen in Seen. - Gas-Wasser-Abrwasser 72: 456-461.

Quny, P. D., Broecker, W. S., Hesslein, E. H., Fee, E. J. \& SCHINDLER, D. W., 1979: Whole lake tritium spikes to measure horizontal and vertical mixing rates. - Isotopes in Lake Studies: 175-194, IAEH, Vienna.

Young, W. R., RHINes, P. B. \& GARRETt, C. J. R., 1982: Shear-flow, internal waves and horizontal mixing in the ocean. - J. Phys. Oceanogr. 12: 515-527.

Authors' address:

Environmental Physics, Swiss Federal Institute of Technology (ETH), Zürich, c/o EAWAG, CH8600 Dübendorf. Switzerland. 